\title{
New Calibration Method of Two-Dimensional Laser Scanner and Camera Based on LM-BP Neural Network
}

\author{
Jianlei Kong, Li Fan, Jinhao Liu ${ }^{*}$, Lei Yan ${ }^{* B}$ and Xiaokang Ding \\ Country College of Technology, Beijing Forestry University \\ No. 35 East Qinghua Road, Haidian District, Beijing, China \\ kongjianlei_slgc@163.com,li_fanfan_1011@163.com,liujinhao@vip.163.com, \\ mark_yanlei@bjfu.edu.cn,dingxiaokang1985@126.com
}

\begin{abstract}
The calibration between a camera and a two-dimensional laser scanner (2DLS) is an essential step in the object detecting system. Many algorithms with linear model have been proposed. But these tend to solve intrinsic and extrinsic calibration parameters separately and are influenced seriously by the poor initial data, which leads to unstable and inaccurate results. Hence, a new nonlinear model based on the Back Propagation neural network trained by the Levenberg-Marquardt algorithm (LM-BP) is presented for calibration in this paper. Before the calibration, the original laser data is fitted linearly to avoid the ranging error and is optimized by an angular increment to reduce the stepangular error. Then, the calibration network with 4 inputs composed of the lasers points' coordinates and constant 1 , and 2 outputs are obtained, expected values of which are the coordinates of corresponding points in the image coordinates. The sum of square of errors between the network outputs and expected values is taken to adjust the modifications of the weights and thresholds with the Levenberg-Marquardt method to optimize the calibration model. Finally, compared with related researches, experimental results show that the accuracy of calibration between camera and 2DLS is significantly improved, and the detecting system is more suitable for actual measurement situations.
\end{abstract}

Keywords: LM-BP neural network, Nonlinear Calibration Model, Two-dimensional Laser, Camera

\section{Introduction}

Two-dimensional Laser scanners (2DLS) and cameras are common sensors used in the reconstruction of three-dimensional environment. A camera can obtain the shape and color of objects effectively by the projection principle. While a laser scanner can not only measure the distance from the device to the object, but also get the relative position between the objects accurately. Therefore, combination of cameras and two-dimensional laser scanners has been widely used to provide applications in the construction industry [1], driver assistance systems [2], aerial mapping [3], military equipment [4], tracking [5] and robots [6].

In order to effectively fuse the data from the camera and 2DLS, it is important to confirm the relative position and orientation between a 2DLS and a camera, which is known as the calibration problem in the 2DLS- camera object detecting system. The calibration between camera and 2DLS is a procedure of determining the intrinsic and extrinsic calibration parameters. The intrinsic parameters include the geometric and optical characteristics of camera and the step-angle and scanning distance in 2DLS. While, the extrinsic parameters are the $3 \mathrm{D}$ position and orientation of the camera frame relative to the 2DLS coordinate system [7]. 
In recent years many scholars have contributed many works on the calibration problem. A new calibration structure of a plane with a triangular hole is used to solve extrinsic calibration parameters in [8]. With this special structure of the calibration plane, the laser scan data is visible in the view of camera, which enhances the efficiency of feature extraction. Some methods focus on optimizing the laser scanner data of the calibration object, which reduces the calibration errors obviously in [9]. However, the extraction process of corresponding data pairs from 2DLS and camera is cumbersome and timeconsuming, which reduces the efficiency in external calibration. What's worse, human intervention causes nonlinear errors and the low accuracy in the calibration process.

To solve those problems, the most widely used method is proposed by Zhang and Pless to describe a 2DLS- camera extrinsic calibration procedure, where a checkerboard pattern is placed at various angles and positions freely in same views of the two sensors simultaneously [10-11]. Assuming that the camera intrinsic parameters are known by using camera calibration method in [12], this method applies the distance vectors perpendicular to the calibration plate to construct homogeneous equations. And the solutions of those equations provide an initial estimate of intrinsic and extrinsic calibration parameters. However, this algorithm ignores the distortion of the camera causing the nonlinear errors. And the specific orientation of calibration plate is placed to obtain the optimal results, which is difficult to be established. Thus, an algorithm has been proposed to establish a coordinate transform relationships by using straight line features in [13]. The method designs a triangular checkerboard to build the constraints equations with the fitting line of the laser points. What's more, a distortion parameter is added to the intrinsic calibration of camera. However, the normal methods above are based on the linear model which doesn't directly enforce the actual nonlinear fitting problem in whole calibration process. And the initial solutions with the high resolution are required in the calibration produce. In addition, those methods are influenced by the poor features corresponding to the laser data and the images. It often leads to the vulnerable stability and the poor noise immunity of the calibration process in the 2DLS-camera system.

In this paper, the Back Propagation neural network trained by Levenberg-Marquardt method (LM-BP) is proposed to solve the calibration problem. In this nonlinear calibration algorithm, the extrinsic calibration matrix in linear methods is fitted via the weights and thresholds between network's output and hidden layers. And the intrinsic matrix is represented by the weights and thresholds between input and hidden layers. Finally the new calibration method optimizes the nonlinear errors and improves the calibration accuracy in the 2DLS-camera system. Display quotations of over 40 words, or as needed.

\section{Linear Model of the 2DLS-camera Calibration}

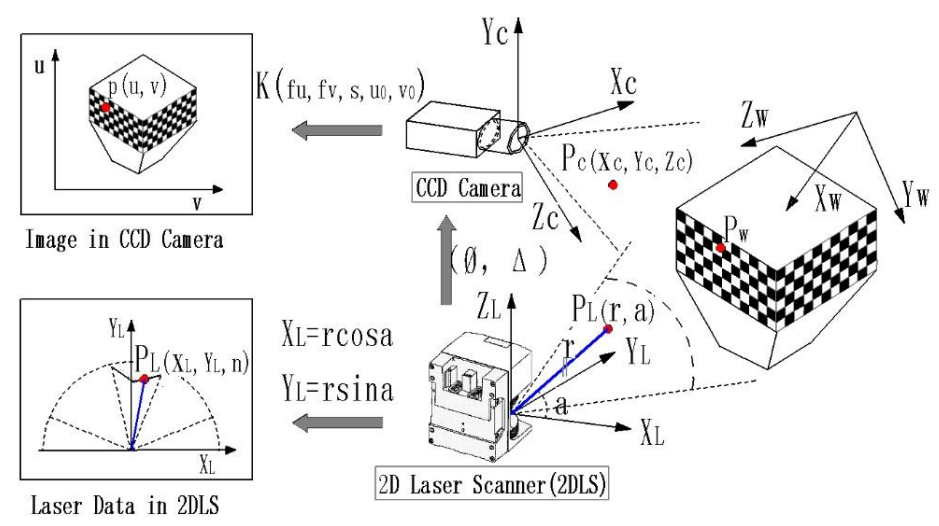

Figure 1. Schematic of the Calibration Process between a Camera and a 2DLS 
The linear calibration model applies the perspective projection principle to determine the internal transformation from the 3D scene to 2D image plane in camera as shown in Figure 1. When an edge point $P_{w}$ of calibration object in the world coordinate system is projected in the camera coordinate system as $P_{c}=\left[X_{c}, Y_{c}, Z_{c}\right]^{T}$, its corresponding projection in the image coordinate system is represented as $p=[u, v]^{T}$. Assuming that the perspective center of camera coincided with the origin of the world coordinate system, the intrinsic calibration parameters inside the camera is obtained by the following transformation formula:

$$
\left[\begin{array}{c}
u \\
v \\
1
\end{array}\right]=c K\left[\begin{array}{c}
X_{c} / Z_{c} \\
Y_{c} / Z_{c} \\
1
\end{array}\right]=\left(c / Z_{c}\right)\left(\begin{array}{cccc}
f_{u} & s & u_{0} & 0 \\
0 & f_{v} & v_{0} & 0 \\
0 & 0 & 1 & 0
\end{array}\right)\left[\begin{array}{c}
X_{c} \\
Y_{c} \\
Z_{c} \\
1
\end{array}\right]
$$

Where $c$ is a constant indicating a proportional factor and $Z_{c}$ represents as the $\mathrm{z}$-axis value of the camera coordinate system. $K$ is the intrinsic matrix related to the camera's internal structure, which is described as $K=\left(\begin{array}{ccc}f_{u} & s & u_{0} \\ 0 & f_{v} & v_{0} \\ 0 & 0 & 1\end{array}\right)$. Here, $f_{u}$ and $f_{v}$ are the equivalent focal lengths on $\mathrm{u}$-axe and $\mathrm{v}$-axe of the image coordinate system respectively. $s$ is the distortion coefficient of camera. $u_{0}$ and $v_{0}$ are the coordinate value of the origin in the image coordinate system.

While, $P_{w}$ is expressed as $P_{L}(r, \alpha)$ in the 2DLS coordinate system and $r$ represents the distance from the object to the laser transmitter. And $\alpha$ is the angle of laser beam in the scanning sector. Here, it is assumed that all laser data is lying on a known scanning plane expressed as $Z_{L}=\eta$, which is parallel to the floor. For ease of calculation, the polar coordinate of $P_{L}$ is converted to the European coordinates as following:

$P_{L}=\left[X_{L}, Y_{L}, Z_{L}\right]=[r \sin (\alpha), r \cos (\alpha), \eta]^{T}$

Since the calibration object is posed in the both views of the camera and 2DLS, the linear calibration model applies the rotation matrix $\Phi$ and the transformed vector $\Delta$ to confirm the extrinsic calibration parameters between a 2DLS and a camera. The relation of them could be described as follows:

$P_{c}=\Phi P_{L}+\Delta$

Where $\Phi$ is a $3 \times 3$ orthonormal matrix and $\Delta$ is a $3 \times 1$ vector represented as $\Delta=\left[\begin{array}{lll}\Delta_{1} & \Delta_{2} & \Delta_{3}\end{array}\right]^{T}$. Combining Eq. (2.1), (2.2) and (2.3), the relation of $p$ and $P_{L}$ lead to the following homogeneous equation:

$$
\left[\begin{array}{c}
u \\
v \\
1
\end{array}\right]=\left(\begin{array}{llll}
M_{11} & M_{12} & M_{13} & M_{14} \\
M_{21} & M_{22} & M_{23} & M_{24} \\
M_{31} & M_{32} & M_{33} & M_{34}
\end{array}\right)\left[\begin{array}{c}
X_{L} \\
Y_{L} \\
Z_{L} \\
1
\end{array}\right]=m\left[\begin{array}{c}
X_{L} \\
Y_{L} \\
Z_{L} \\
1
\end{array}\right]
$$

Here $m$ is called as projection matrix between a camera and 2DLS, and the solution seeking of $\mathrm{m}$ concludes the initial rotate matrix $\Phi$ and the transformed vector $\Delta$ in extrinsic calibration of 2DLS-camera. Usually the Levenberg-Marquardt method is used to refine calibration parameters by iterative minimization of the re-projection error in the linear calibration model. 


\section{Calibration Model Based On LM-BP Neural Network}

\subsection{Laser Data Improvement}

Before designing the network, two common problems existing in laser data extracting should be optimized to improve the calibration accuracy greatly. The emitted laser beams are deflected using an internal rotating mirror at regular steps and scanning the surroundings in a circular manner. This leads that the laser data standing for edge points may be far away from the actual edges of objects, which causes the step-angular error and reduces the calibration accuracy. In addition, the distance to the object is calculated by the time required for the pulsed beam to be reflected and received by the sensor. Since the reflection of laser beams would vary as a function of the surface's structure and color, the distances detected by the same laser beam are discriminative in different moments. This situation will cause the ranging error. To reduce the detecting errors between the acquired laser data and the real world feature, an optimized method is proposed to reduce the ranging error and the step angular error as shown in Figure.2.

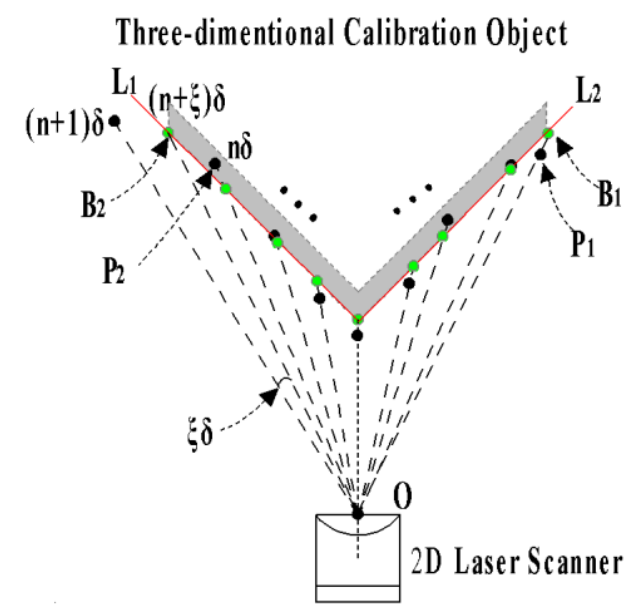

\section{Figure 2. Schematic of Laser Data Improvement}

A black dot $P_{1}$ deviated from the calibration object surface indicates the actual measurement of the laser points. And the green point $B_{1}$ represents the adjusted laser data corresponding to $P_{1}$. This work applies the method of least squares to linearly fit laser points of the calibration object, which is captured by 2DLS as intersecting lines $L_{1}$ and $L_{2}$ in 2DLS coordinate system. The lines are expressed as:

$L_{i}=\left\{\left(\sum_{k=1}^{m} x_{k}, \sum_{k=1}^{m} y_{k}\right) \mid \sum_{k=w}^{m} y_{k}=a_{i} \sum_{k=w}^{m} x_{k}+b_{i} ; i=1,2\right\}$

Where $X_{k}$ and $Y_{k}$ represents the coordinate of the k-th feature points and satisfies the constraint $D_{k}=\sqrt{X_{k}^{2}+Y_{k}^{2}}$, Here $D_{k}$ is the distance from the point on the object to the origin of laser sector. The parameters $a$ and $b$ represent the slopes and the intercepts of lines respectively, which are obtained as following:

$a_{i}=\frac{m \cdot \sum_{k=1}^{m} x_{k} y_{k}-\sum_{k=1}^{m} x_{k} \sum_{k=1}^{m} y_{k}}{m \cdot \sum_{k=1}^{m} x_{k}^{2}-\left(\sum_{k=1}^{m} x_{k}\right)^{2}} ; b_{i}=\frac{\sum_{k=1}^{m-1} y_{k}-a_{i} \sum_{k=1}^{m-1} x_{k}}{w-1} ;(i=1,2)$ 
Combining Eq. (3.1) and (3.2), the optimized laser points (green dots in Figure 2) are used to reduce the ranging error in calibration process. Since the edge point $P_{2}=(n \delta, r)$ detected by 2DLS is not on the edge of object accurately due to the angular resolution $\delta$, a nearby estimated point $B_{1}$ is used to replace $P_{2}$ by bisecting the included angle of the neighbouring laser beams. Here $\xi$ is the bisected parameter in the range between 0 and 1 . So the coordinate of optimized point $B_{1}=(n \delta+\xi \delta, r)$ is used to reduce the step-angular error, which improves greatly the accuracy of calibration in 2DLS-camera system.

\subsection{Design of LM-BP Neural Network}

Back propagation neural network (BP) is a multi-forward type networks and belongs to the supervised learning algorithms. When the signals are propagated forward, the error of network is propagated back in BPNN. Using the gradient descent algorithm to train the network, the weights and thresholds amongst layers of neurons are continuously adjusted to the minimum mean-square error between the actual output and the desired output. Because of the good performance in adaptability and effectiveness, the BPNN has been widely used in single camera calibration [13], Stereo Camera calibration [14] and multisensor fusion [15].

Therefore, a LM-BP neural network is adopted to the 2DLS-camera detecting systems. The network has 3 layers, whose input layer has 4 neurons, it's signals are composed of laser points' coordinates in 2DLS coordinate system and constant 1 . And the hidden layer has 3 neurons and output layer has 2 neurons desiring the coordinates of corresponding point in the image coordinates. The network structure is shown in Figure 3.

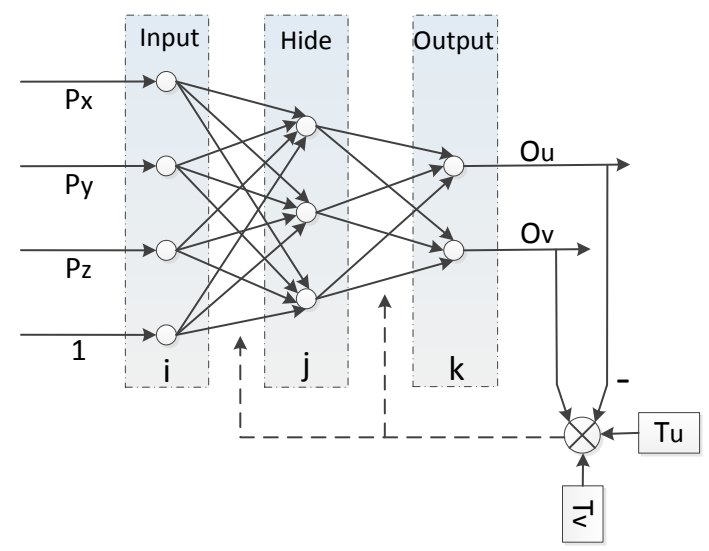

\section{Figure 3. Structure of LM-BP Neural Network}

The weights and thresholds between input layer and hidden layer could be taken as $3 \times$ 4 matrix, which are elements of fitted extrinsic calibration matrix. And weights and thresholds between hidden layer and output layer are taken as $2 \times 3$ matrix which fitted the intrinsic matrix $\mathrm{K}$ of the camera. This structure adjusts the weights and thresholds dynamically to calculate intrinsic and extrinsic calibration parameters in 2DLS-camera system simultaneously. This method conforms to physical sense and optimizes the nonlinear errors globally, which improves the calibration accuracy. The $\mathrm{j}$-th neuron's inputs in the hidden layer are :

$N e t_{j}=\sum_{i} w_{i j} L_{i}-\theta_{j} \quad(i=1,2,3,4 ; j=1,2,3)$ 
Where $L_{i}$ is the coordinates of feature point. $w_{i j}$ is the weight between the input layer and hidden layer and $\theta_{j}$ is the corresponding threshold. Let the activation function $\mathrm{f}(\mathrm{x})$ be $\mathrm{S}$ tangent function. Thus the outputs of $\mathrm{j}$-th neuron's in the hidden layer are:

$H_{j}=f\left(\right.$ Net $\left._{j}\right) \quad(j=1,2,3)$

Similarly the k-th neuron's inputs in the output layer are:

$N e t_{k}=\sum_{j} w_{j k} H_{j}-\theta_{k} \quad(j=1,2,3 ; k=u, v)$

Here $w_{j k}$ is the weight and $\theta_{k}$ is the threshold between the hidden layer and output layer. And the activation function $f(x)$ is chosen as the linear function. So the $k$-th neuron's output in the output layer is shown as follows:

$O_{k}=f\left(\right.$ Net $\left._{k}\right) \quad(k=u, v)$

During the learning, the desired values of the network are $T_{\mathrm{u}} 、 T_{\mathrm{v}}$, which are the projected pixel coordinates of laser points. The sum of square of errors between actual outputs of network and desired value is shown as follows:

$E=\frac{1}{2} \sum_{k=u, v}\left(T_{k}-O_{k}\right)^{2}=\frac{1}{2} \sum_{k=u, v}\left\{T_{k}-f\left[\sum_{j=1}^{3} w_{j k} f\left(\sum_{i=1}^{4} w_{i j} L_{i}-\theta_{j}\right)-\theta_{k}\right]\right\}^{2}$

While the performance of $E$ comes to minimum, the weights and thresholds among the network are tuned via errors back propagating in light of gradient descent rule. Let the gradient between the hidden layer and the output layer be: $\delta_{k}=-\frac{\partial E}{\partial w_{j k}}\left(T_{k}-O_{k}\right) \square f\left(\mathrm{Net}_{k}\right)^{\prime}$. Then the modifications of $w_{j k}$ and $\theta_{k}$ are obtained as following:

$\Delta w_{j k}=-n \frac{\partial E}{\partial w_{j k}}=N \delta_{k} H_{j} ; \Delta \theta_{k}=N \frac{\partial E}{\partial \theta_{k}}=-N \delta_{k}$

At the same time, a gradient is introduced to the weights $w_{i j}$ and the thresholds $\theta_{j}$ between the input layer and hidden layer as $\delta_{j}=\left(\sum \delta_{k} w_{i j} \backslash \square f\left(\text { Net }_{j}\right)^{\prime}\right.$. Similarly the corresponding modifications are obtained as following:

$\Delta w_{i j}=N \delta_{j} L_{i} ; \Delta \theta_{j}=-N \delta_{j}$

In order to enhance the classical algorithm of BP neural network, the method of Levenberg-Marquardt(L-M) is applied to optimize the modifications of weights and thresholds comprehensively. The L-M algorithm applies a nonlinear least squares method to deduce the approximate form of Hessian matrix, which accelerates the convergence rate of network and prevented the local minimization problem of calibration process greatly. Let the correction of $\Delta w_{j k}$ and $\Delta \theta_{k}$ be $\Delta x_{j k}$. Similarly let the correction of modifications between the input layer and the hidden layer be $\Delta x_{i j}$ as following:

$$
\begin{aligned}
& \Delta x_{j k}=N\left[J^{T}\left(x_{1}\right) J\left(x_{1}\right)+\mu I\right]^{-1} J\left(x_{1}\right)^{T} \mathrm{e}\left(\mathrm{x}_{1}\right) \\
& \Delta x_{i j}=N\left[J^{T}\left(x_{2}\right) J\left(x_{2}\right)+\mu I\right]^{-1} J\left(x_{2}\right)^{T} e\left(x_{2}\right)
\end{aligned}
$$


Where $x_{1}=\left[w_{l l}^{l}, \cdots, w_{i j}^{l}, \theta_{1}^{l}, \cdots, \theta_{k}^{l}, w_{1 l}^{2}, \cdots, w_{i j}^{2}\right], x_{2}=\left[w_{l l}^{l}, \cdots, w_{i j}^{l}, \theta_{1}^{l}, \cdots, \theta_{j}^{l}, w_{1 l}^{2}, \cdots, w_{i j}^{2}\right]$ and $\mathrm{e}(\mathrm{x})$ is inputs of every layer. Here $\mathrm{J}(\mathrm{x})$ is the Jacobian matrix and $\mathrm{I}$ is a unit vector. $\mathrm{N}$ is the learning step of the network and $\mu$ is a non-negative coefficient. The calculation procedure would be found in [17]. Finally these weights and thresholds can be calculated in LM-BP neural network as following:

$$
\begin{aligned}
& w_{j k}(n+1)=w_{j k}(n)+\Delta x_{j k} ; \theta_{k}(n+1)=\theta_{k}(n)+\Delta x_{j k} \\
& w_{i j}(n+1)=w_{i j}(n)+\Delta x_{i j} ; \theta_{j}(n+1)=\theta_{j}(n)+\Delta x_{i j}
\end{aligned}
$$

In calibration process, input signals are composed of constant 1 and laser points' coordinates, which should be optimized by the method above. And outputs $\left[O_{v}, O_{u}\right]$ are obtained through network. Let the sum of square errors $E=0.5\left[\left(T_{u}-O_{u}\right)^{2}+\left(T_{v}-O_{v}\right)^{2}\right]$ between the outputs and deserved vector $\left[T_{u}, T_{v}\right]$ as performance index to check whether or not the performance index meets the expected requirement $\varepsilon$. If not, the weight and the threshold matrix are tuned and trained by the L-M method to obtain the modifications, which corrects the LM-BP neural network iteratively. If all the training samples less than 1000 are over, the next round training begins again until the performance index is reached, and the weight and threshold matrix converges to stable value. Finally, the calibration process in the model of LM-BP neural network is finished. The flow chart of program is illustrated in Figure 4.

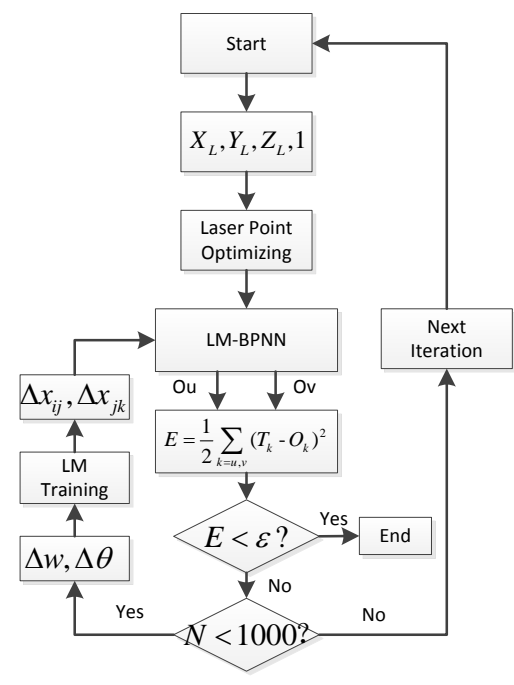

Figure 4. Flowchart of Calibration Program

\section{Experiments}

\subsection{Comparison}

The paper designs a three-dimension calibration object, which is placed at the distance ranging from 2 meter to 6 meter in calibration experiments. And this experiment deals with the effect on the calibration performance caused by number of calibration object poses and orientations. Thus the average errors are calculated by running 150 trials with 15 positions. And the orientation angles of calibration object vary from 0 to 90 at every position.

The establishment of LM-BP networks and the calibration process has been implemented in Matlab. Set the learning step of neural network be 0.3 and the display 
interval of training be 2000. Let the largest number of training be 10000 and the minimum mean square error be 0.00001 . What's more, block Hessian matrix calculation is set as 10 and the maximum training time is limited to 10 seconds. Let the activation function between the input layer and hidden layer be the tansig function and the activation function between the hidden layer and output layer be the purelin function in MATLAB. Finally, the trainlm function is selected as the training function in MATLAB to optimize the BP neural network. The weight and threshold between the input layer and hidden layer are calculated as:

$$
W_{i j}=\left[\begin{array}{cc}
0.1151 & -0.3867 \\
0.3395 & 0.2457 \\
0.4416 & 0.4676
\end{array}\right], \theta_{j}=\left[\begin{array}{lll}
-1.0112 & -0.3813 & -0.8834
\end{array}\right]^{T}
$$

While the weight and threshold between the hidden layer and output layer are obtained as:

$$
W_{j k}=\left[\begin{array}{ccc}
2.6677 & 4.7040 & -2.4896 \\
5.6151 & -1.7110 & 1.3066
\end{array}\right], \theta_{k}=\left[\begin{array}{ll}
1.8878 & 4.2703
\end{array}\right]^{T}
$$

In order to compare with the linear methods proposed by Zhang(2004) in [10] and the method proposed by $\mathrm{Li}(2004)$ in [13], the 95 sets of experimental data are selected as the same inputs to compute the different calibration models, and the other 40 sets of experimental data are chosen as testing data to evaluate the calibration performance. Then several contrast parameters are selected to verify high accuracy of calibration with the new algorithm as showed in Table 1.

\section{Table 1. Experimental Result of New Algorithm Compared with Related} Works

\begin{tabular}{llll}
\hline Methods & Zhang (2004) & Li(2007) & LM-BPNN \\
\hline Root-Mean-Square Error(RMSE) & 10.232 & 10.096 & 5.123 \\
Standard Deviation(STD) & 19.823 & 15.027 & 13.032 \\
u-axis average error(pixel) & 19.823 & 18.216 & 14.535 \\
v-axis average error(pixel) & 43.532 & 35.810 & 17.473 \\
Three-dimensional error in 6 meters (cm) & 5.819 & 4.859 & 2.764 \\
Angle error in 6 meters(degree) & 1.164 & 0.972 & 0.553 \\
\hline
\end{tabular}

As illustrated in Table 1, this method works on the best effect on various indicators compared with the linear methods. The Root-Mean-Square Error (RMSE) of the calibration model in LM-BP neural network is closed to 5.123, which is obviously improved by $49.93 \%$ compared with Zhang $(2004)$ and by $49.23 \%$ corresponding to $\mathrm{Li}(2007)$. The best performance in RMSE indicates that the new calibration method reduces the deviation between the feature points and the corresponding projections and improved the precision of 2DLS-camera system. Moreover, the LM-BPNN obtained the minimum value in the Standard Deviation (STD) of the calibration errors as 13.032, which has a huge improvement at $34.26 \%$ compared with Zhang(2004) and at $13.28 \%$ corresponding to $\mathrm{Li}(2007)$. It means that the discrete degree of the new model is very lower, which leads to good stability and robustness in calibration process.

What's more, this paper applies higher resolution images to calibration procedure compared with the experiments in Zhang(2004) and $\operatorname{Li}(2007)$. And the new method relegate the u-axis and v-axis average error to 14.535 (pixel) and 17.473 (pixel) 
respectively, which both are the minimum value relative to linear calibration models. Those evaluation indexes indicate that the new algorithm has a huge improve in the calibration accuracy. Here the calibration object is placed at the distance, which ranged from $2(\mathrm{~m})$ to $6(\mathrm{~m})$ in this contrastive experiment. When the distance meets the farthest value, the three-dimensional error in this algorithm is about in $\pm 0.1(\mathrm{~cm})$ around 2.764 $(\mathrm{cm})$, which is less than the error about $5.819(\mathrm{~cm})$ obtained by Zhang $(2004)$ and 4.859 (cm) corresponding to $\operatorname{Li}(2007)$. Similarly, the value of angle error in this new algorithm obtains the smallest value about 0.553 (degree) in world coordinate system. It presents the high precision and accuracy in calibration process with the new methods. Hence the LMBP neural network method is more suitable for the actual detecting system and is less affected by the distance and position.

Further experiment dealing with the influence of the number of data pairs on the calibration error is performed. The number of the data sets is varied from 35 to 140, and the calibration error of every number is computed by running three methods as presented in Figure 5. Where x-axis represents the number of the data pairs and y-axis represents the calibration error. In general, the three-dimensional error curve of actual calibration with the new algorithm is lower than Zhang(2004) and $\operatorname{Li}(2007)$, which indicates a higher calibration accuracy and stability in calibration produce. The error curve of the new method reaches a stable value at about at 70 sets and the ones in the other methods tend to smooth at 75 sets. It could be seen that the new method has higher rate in iterative convergence and is faster to reach steady state in calibration progress. And all error curves shock between 70 sets and 120 sets and achieve the minimum at 95 sets. To reduce the negative effects caused by number of data pairs on actual three-dimensional error, the 95 sets should be chosen as the optimal number in the calibration experiment.

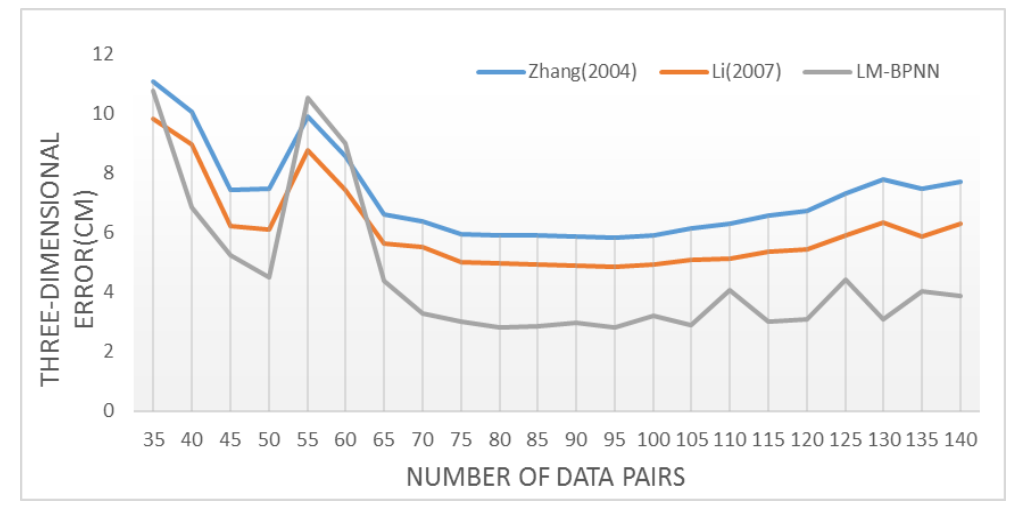

\section{Figure 5. The Influence of the Number of Data Pairs on the Three- Dimensional Error}

Since the calibration object is placed in different angles and positions, another experiment is performed to reveal the influence of the angles and positions on the orientation error as showed in Figure 6. Here x-axis represents the number of poses, which are located in various angles and positions. And the y-axis represents the orientation error. The values of the error curve with linear models are higher than the ones in LM-BPNN, which show that the new method has higher veracity in orientation error. What's more, the curve with the new method is stable in the range 65 and 130, and the stable segment of the error curve in Zhang (2004) and Li (2007) is between 75 and 105, which indicated that the new calibration algorithm has a longer range of stability and adapt to the physical environment excellently. And it also shows that the orientation errors with the new algorithm are weakly affected by the angles and positions of the calibration object. Whereas, the results obtained by Zhang (2004) and L i(2007) are strongly influenced by number of poses. Therefore, it can be concluded that the 
calibration model of LM-BPNN could reduce the calibration errors and is optimal in terms of noise immunity, which is suitable for the actual applications.



Figure 6. The Influence of the Number of Poses on the Orientation Error

\subsection{Experiments with Real Data}

Experiments are carried out on the equipment as shown in table II. Compared with images of $640 \times 480$ (pixel) viewed by a DFW-VL500 camera in Zhang(2004) and ones of $1024 \times 768$ (pixel) taken with a UNIQ UC-800 camera in Li(2007), this work applies a CANOY EOS550D SLR camera to take $2592 \times 1728$ (pixel) images, which present higher resolution and larger magnitude of the average errors in calibration procedure corresponding to relative works. And a SICK LMS511-20100 laser scanner provides maximum $80(\mathrm{~m})$ measurements with 0.176 (degree) angular resolution by scanning 190(degree) parallel to the floor, which offeres a higher precision laser data than the 2DLS in Zhang(2004) and $\operatorname{Li}(2007)$ and reduces the calibration errors. A rectangular calibration-object with two checkerboard patterns, which is defined by $30 \times 30$ (mm) grids, is applied to the calibration progress. The design of the calibration object is beneficial to calculation of intrinsic and extrinsic parameters with linear calibration models simultaneously in 2DLS-camera detecting system. Here the object is placed in the distance ranging from 2 meter to 6 meter, which is farther than the experimental distance of a single checkerboard in $\operatorname{Zhang}(2004)$ and $\mathrm{Li}(2007)$. And the calibration experiments have been carried out 150 times comparing to 100 trials in normal methods. Finally, the three-dimensional error of the new method is about in $\pm 0.1(\mathrm{~cm})$ around $2.76(\mathrm{~cm})$, which is more accurate and stable than the result in Zhang(2004) and $\mathrm{Li}(2007)$.

Table 2. The Parameters for the Performances

\begin{tabular}{llll}
\hline Methods & Zhang (2004) & Li(2007) & LM-BPNN \\
\hline Camera Model & DFW-VL500 & UNIQ UC-800 & CANOY EOS550D SLR \\
2DLS Model & SICK PLS & SICK LMS221-30206 & SICK LMS511-20100 \\
Image Size(pixel) & $640 * 480$ & $1024 * 768$ & $2592 * 1728$ \\
Distance(m) & 1.5 & 1.5 & $2-6$ \\
Pose(trails) & 100 & 100 & 150 \\
Time(s) & 0.050041 & 0.038693 & 0.539515 \\
\hline
\end{tabular}

To demonstrate superior performance of the new method over the normal methods in $\mathrm{Li}(2007)$, the measurements of the 2DLS are projected onto the corresponding images of camera using calibration parameters of both methods. The results are displayed in Figure 7. The lower solid lines (green) represent the projections of the LRF measurement using the parameters calibrated by the new method, and the upper dotted lines (blue) denote 
those obtained by the method in $\operatorname{Li}(2007)$. As observed obviously, the projected points obtained by LM-BPNN method more fitted the scene in the two images. Therefore, the new algorithm has the better performance in calibration of 2DLS-camera.

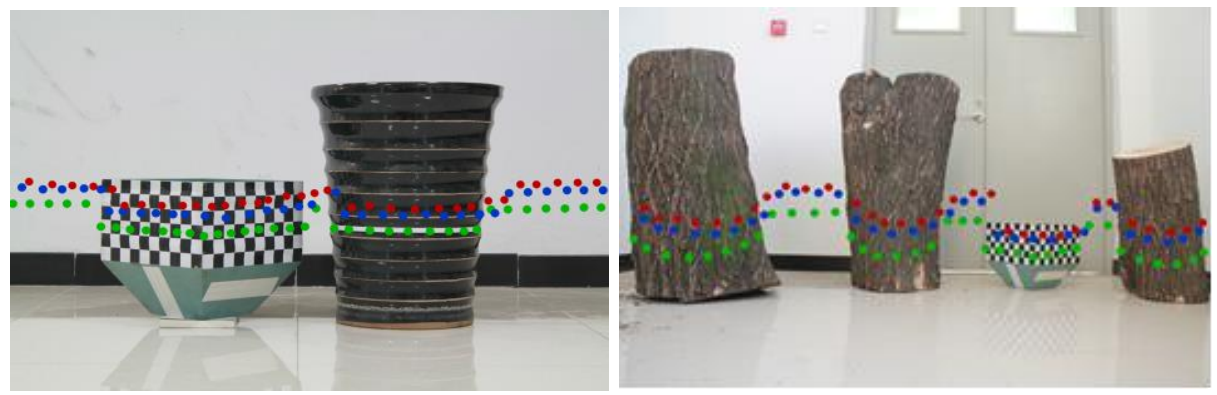

\section{Figure 7. Projection of the Laser Points into the Images with the New Method (Green), the Method in Zhang (Red) and the Method in Li (Blue)}

\section{Conclusions}

In this paper, a new nonlinear model based on LM-BP neural network is proposed to improve accuracy of calibration between camera and 2DLS. The network structure is designed according to linear calibration model, where there are 4 inputs composed of the sampled points' coordinates in 2DLS coordinate system and constant 1, and 2 outputs are obtained, expected values of which are the coordinates of corresponding point in the image coordinates. The sum of square of errors between the network outputs and expected values is taken to adjust the weights and thresholds in light of the gradient descend rule. Then, the Levenberg-Marquardt method is applied to train the network by optimizing the modifications of weights and thresholds. Here, the original laser data is fitted linearly to avoid the ranging error and is optimized by the angular increment to reduce the stepangular error, which improved the accuracy of laser data.

Although the computation speed of new method is relatively slower than normal methods, but the value is not great and did not affect the actual application. And comparing with Zhang(2004) and $\operatorname{Li}(2007)$, the LM-BPNN algorithm has better performance in calibration accuracy and system stability. Experimental results show that the three-dimensional error is down to about $0.1(\mathrm{~cm})$ around $2.764(\mathrm{~cm})$ at $6(\mathrm{~m})$ distance and the angular error is down to about 0.553(degree). Moreover, the new method obtain better stability and robustness than related works in calibration process, which makes the 2DLS-camera detecting system more suitable for actual measurement situations.

\section{Axknowledgements}

This work is partially based on the 948 introduced project, automatic measurement technology equipped on forestry harvesting machines supported by the Chinese State Forestry Bureau (Grant No. 2011-4-02).This study is also financially supported by Beijing Higher Education Young Elite Teacher Project (YETP0759), China Postdoctoral Science special Foundation (2013T60070).

\section{References}

[1] B. Yunsu, C. Donggul, J. Yekeun and I. S. Kweon, "Capturing village-level heritages with a hand-held camera-laser fusion sensor", International Journal of Computer Vision, vol. 94, no. 1, (2011), pp. 36-53.

[2] N. Kämpchen, "Feature level fusion of laser scanner and video data for advanced driver assistance systems", Univ., Fak. für Ingenieurwiss. und Informatik, (2007).

[3] M. Whalley, G. Schulein and C. Theodore, "Design and flight test results for a hemispherical ladar developed to support unmanned rotorcraft urban operations research", ANNUAL FORUM PROCEEDINGS-AMERICAN HELICOPTER SOCIETY. AMERICAN HELICOPTER SOCIETY, INC, 
vol. 64, no. 2, (2008), pp. 1672.

[4] C. Rasmussen, "Combining laser range, color, and texture cues for autonomous road following, Robotics and Automation, 2002. Proceedings. ICRA'02. IEEE International Conference, vol. 4, (2002), pp. 43204325.

[5] J. Cui, H. Zha, H. Zhao and R. Shibasaki, "Multi-modal tracking of people using laser scanners and video camera", Image and vision Computing, vol. 26, no. 2, (2008), pp. 240-252.

[6] V. Pradeep, K. Konolige and E. Berger, "Calibrating a multi-arm multi-sensor robot: A bundle adjustment approach, Experimental Robotics”, Springer Berlin Heidelberg, (2014), pp. 211-225.

[7] Q. V. Le and A. Y. Ng, "Joint calibration of multiple sensors", In Intelligent Robots and Systems, IROS'09. IEEE/RSJ International Conference, (2009), pp. 3651-3658.

[8] J. E. Ha, "Extrinsic calibration of a camera and laser range finder using a new calibration structure of a plane with a triangular hole", International Journal of Control, Automation and Systems, vol. 10, no. 6, (2012), pp. 1240-1244.

[9] S. Krause and R. Evert, "Remission based improvement of extrinsic parameter calibration of camera and laser scanner", In Control Automation Robotics \& Vision (ICARCV), IEEE 12th International Conference, (2012), pp. 829-834.

[10] Q. Zhang and R. Pless, "Extrinsic calibration of a camera and laser range finder", (improves camera calibration) In Intelligent Robots and Systems, IROS'04, IEEE/RSJ International Conference, vol. 3, (2004), pp. 2301-2306.

[11] Q. Zhang and R. Pless, "Constraints for heterogeneous sensor auto-calibration. In Computer Vision and Pattern Recognition Workshop”, CVPRW'04 IEEE Conference, (2004), pp. 38-38.

[12] Z. Zhang, "A flexible new technique for camera calibration. Pattern Analysis and Machine Intelligence", IEEE Transactions, vol. 22, no. 11, (2000), pp. 1330-1334.

[13] G. Li, Y. Liu, L. Dong, X. Cai and D. Zhou, "An algorithm for extrinsic parameters calibration of a camera and a laser range finder using line features", In Intelligent Robots and Systems, IROS'07, IEEE/RSJ International Conference, (2007), pp. 3854-3859.

[14] D. Y. Ge and X. F. Yao, "Camera calibration and precision analysis based on BP neural network", In Image and Signal Processing, CISP'09, 2nd International Congress, pp. 1-5.

[15] J. L. Xiong, J. Y. Xia, X. Q. Xu and Z. Tian, "A Novel Method of Stereo Camera Calibration Using BP Neural Network. Applied Mechanics and Materials, vol. 29, (2010), pp. 2692-2697.

[16] P. Chang and J. S. Shih, "The application of back propagation neural network of multi-channel piezoelectric quartz crystal sensor for mixed organic vapours", Tamkang Journal of Science and Engineering, vol. 5, no. 4, (2002), pp. 209-218.

[17] M. T. Hagan and M. B. Menhaj, "Training feedforward networks with the Marquardt algorithm", Neural Networks. IEEE Transactions, vol. 5, no. 6, (1994), pp. 989-993.

Authors

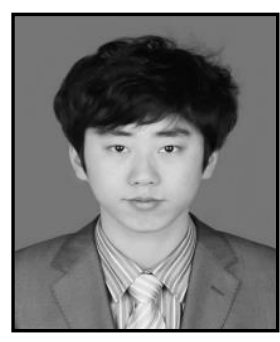

Jianlei Kong, Received his B.Sc. degree in Robotization Engineering from Beijing Forestry University in 2011. During 2011 and 2014, he received M.Sc of the Forest Engineering from Country College of Technology in Beijing Forestry University. Now he is a $\mathrm{Ph} . \mathrm{D}$. candidate in Research Institute of Forestry Vehicle and special Equipment. His research lies in the fusion of mutli-sensors including computer vision and laser scanners, focusing on 3D reconstruction and pattern recognition. In the past two years, he has been working on efficient algorithms to construct 3D coloured models from largescale point clouds and visual patterns. Currently, his research focus shifts to develop intelligent systems that recognize scenes and obstacles for forestry mapping and autonomous navigation of harvesters..

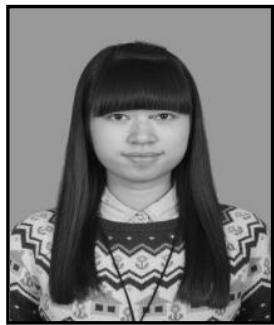

Li Fan, Received her B.Sc. degree in Vehicle Engineering from Beijing Forestry University in 2013. Now she is a M.Sc. candidate in Research Institute of Forestry Vehicle and special Equipment. Her research focus is 3D point cloud data and pattern recognition. In the past two years, she has been working on filtering and feature extraction of 3D point cloud data. Currently, she is working on efficient algorithms to classify 3D point cloud. 


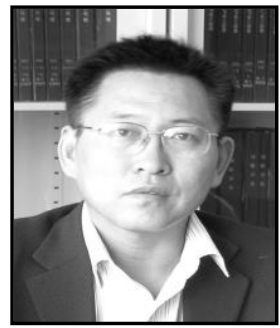

Jinhao Liu, Received the Ph.D. degree in Forestry engineering from the Northeast Forestry University, China, in 1994. He is currently Professor of Forestry engineering at Beijing Forestry University, China. $\mathrm{He}$ is the director of the Forestry and Environmental special equipment laboratory, Beijing Forestry University, China. He was the deputy-secretary general of the Packaging Education Committee of Chinese Packaging Federation. And $\mathrm{He}$ also holds an appointment as vice chairman of Forestry Engineering Committee of Chinese Forest Society Committee. His research covers design and manufacture for mechanical system, forestry special robots, harvesting and transport of woods in forest, and forestry machinery and equipment. And He has led four "863" projects and invented trees cone collecting robot, the new smart stump cleaning robot, intelligent centering on the peeling wooden robot, stereo sand-fixing car. Most recently he has been developing and researching multifunctional forestry felling \& cultivation machine in national "Eleventh five-Year "Science and Technology support project.

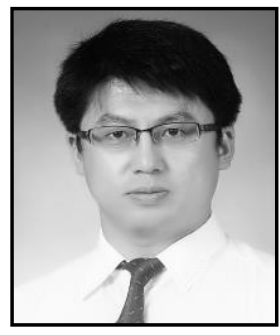

Lei Yan, Is now associate professor of Beijing Forestry University engineering automation system. In July 2004, he receives master degree in mechanical and electrical engineering, Jilin University. In August 2010, graduated from South Korea north gyeongsang national university, received a doctor's degree in engineering. Then in Beijing Forestry University, institute of technology, is engaged in the automatic control system, image acquisition and processing system development, signal processing and pattern recognition algorithm of research work. He has published more than 50 papers, of which includes $6 \mathrm{SCI}$ articles, more than 20 EI papers. He also applies for more than 20 patents for invention and a total of 15 software copyright with authorized software copyright. He is As a Scientific Reports of ASME the Transactions on Mechatronics review.

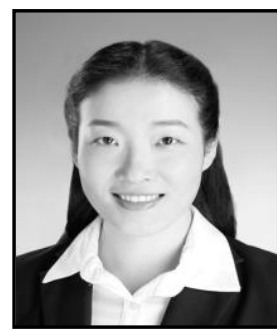

Xiaokang Ding, Received her B.Sc. degree in Electricity Information and Technology from Beijing Forestry University in 2008. And received M.Sc degree in Biophysics from Beijing Forestry University in 2012. Now she is a Ph.D. candidate in Forestry vehicle and special equipment group. Her research lies in the fusion of mutlisensors including computer vision and laser scanners, focusing on pattern recognition. 
International Journal of Signal Processing, Image Processing and Pattern Recognition Vol. 9, No. 7 (2016) 